\title{
Robust Optimal Nonlinear Control with Observer for Position Tracking of Permanent Magnet Synchronous Motors
}

\author{
Dong-Hyun $\mathrm{Ha}^{*}$, Chang-Soon Lim ${ }^{* *}$, and Dong-Seok Hyun ${ }^{\dagger}$ \\ "Division of Electrical and Computer Engineering, Hanyang University, Seoul, Korea \\ "Hyundai Rotem, Uiwang, Korea \\ ${ }^{* * \dagger}$ Dept. of Electrical Engineering, Hanyang University, Seoul, Korea
}

\begin{abstract}
This paper proposes a robust optimal nonlinear control with an observer to reject the offset errors of position tracking for surface mounted permanent magnet synchronous motors. We provide the control method to reject offset errors and load torque for designing field oriented control (FOC) based the alternating current (AC) frame. The proposed method consists of a torque generator, a commutation scheme, an electrical controller, and a load torque observer. The mechanical controller is designed to compensate for load torque and the offset error and generate the desired torque. The commutation scheme is proposed to create the desired currents for the desired torque. The electrical controller is developed to guarantee the desired currents. The observer is designed to estimate both the velocity and the load torque. In order to obtain the robustness to parameter uncertainties and a gain tuning guide, the linear quadratic regulator method is applied to the proposed method. The closed-loop stability is proven. A detailed process for the FOC design and an analysis of the control methods based on the AC frame are presented. The performance of the proposed method was validated via experiments. The proposed method obtains the FOC based on the AC frame. Furthermore, the position tracking performance of the proposed method is superior to that of the conventional method.
\end{abstract}

Keywords: DQ transformation, Permanent magnet synchronous motor, Position control

\section{INTRODUCTION}

Permanent magnet synchronous motors (PMSMs) have been widely used in variable frequency drive applications because they have several advantages over commonly used motors i.e., the absence of external rotor excitation, high power density, and a rapid dynamic response due to a high torque-to-inertia ratio [1]-[3]. Therefore, PMSMs cover the largest part of servo applications.

To improve the performance of the PMSM, various nonlinear control methods based on field oriented control (FOC) have been developed [4]-[7]. A control design for a PMSM was derived from input-output linearization [4]. The input-output linearization made it possible to write the PMSM's

Manuscript received Jun. 12, 2013; revised Sep. 11, 2013

Recommended for publication by Associate Editor Sanjeet K. Dwivedi.

CorrespondingAuthor:dshyun@hanyang.ac.kr

Tel:+82-2-2220-0345,Fax:+82-2-2220-0532, Hanyang University

*Division of Electrical and Computer Engineering, Hanyang University,

Korea

${ }^{*}$ Hyundai Rotem, Korea

${ }^{* *}$ Dept. of Electrical Engineering, Hanyang University, Korea model in the Brunovski decoupled canonical form which makes the synthesis of linear controllers possible. A digital signal processor (DSP)-based robust nonlinear speed control based on input-output linearization was presented [5]. A method of design optimization for the minimization of force ripples and the maximization of the thrust force in brushless permanent magnet motor was represented [6]. The current control loops were implemented using a PI current controller in order to present a solution for velocity control in PMSMs [7]. It was proven that velocity converges to a constant desired value from any initial condition. In motor system operation, the constant disturbance may appear due to load torque or sensor error [8]. Although the previous methods improved the performance of PMSMs, the offset error due to constant disturbances of both the mechanical and electrical systems in PMSMs were not considered. There is no gain tuning guide to obtain control performance. Furthermore, the previous methods required the direct-quadrature (DQ) transformation to analyze PMSMs based on the direct current (DC) frame. Essentially, PMSMs operate based on the alternating current (AC) frame. Thus, a detailed process for FOC design or analysis of the 
control methods based on the AC frame is required. Recently, a two phase frame based nonlinear controller was proposed to improve the position control for PMSMs [9], [10]. However, in that paper, the effects of the load torque and disturbances were not considered. Furthermore, the previous methods require velocity and load torque information. However, it is difficult to measure both velocity and load toque due to the limitations of space and/or cost. In [11], [12], observers were proposed to estimate the position or load torque without the DQ transformation. However, the controllers were designed with DQ transformation. Another problem of the previous nonlinear control methods is that there are no gain tuning guides. Recently, control design methods based on linear quadratic regulators (LQRs) have been designed to achieve robust stability and performance despite model inaccuracies for the applications of DC motors and converters [13], [14].

This paper proposes a robust optimal nonlinear control with an observer to compensate for constant disturbances and load torque based on the AC frame for position tracking of PMSMs. The offset error due to sensor offset errors and the load torque are the main causes of performance degradation in PMSMs. The proposed method consists of a torque generator, a commutation scheme, an electrical controller, and a load torque observer. The mechanical controller is designed to compensate for the load torque and the offset error and to generate the desired torque. The commutation scheme is proposed to make the desired currents for the desired torque. We provide a method for designing FOC based on a two-phase frame. The electrical controller is developed to guarantee the desired currents. Finally the velocity and load torque observer is designed to estimate both the velocity and load torque. In order to obtain the robustness to parameter uncertainties and the gain tuning guide, the controller gain of the proposed method is determined by the LQR method. The closed-loop stability is proven. The detailed process for the FOC design and the analysis of the control method based on the AC frame is presented. The performance of the proposed method was validated via experiments. The proposed method obtains the FOC based on the AC frame. Furthermore, the position tracking performance of the proposed method is superior to that of the conventional method.

This paper is organized as follows. The mathematical model of the PMSM and the conventional control methods are reviewed in Section II. The controller design is detailed in Section III. The experimental results are presented in Section IV. Conclusions are given in Section V.

\section{MODEL AND REVIEW OF ClASSICAL CONTROL METHODS}

\section{A. Mathematical Model of Surface Mounted PMSM}

The three phase PMSM model can be transformed into the $\alpha-\beta$ frame model using the Clarke transformation [1].

$$
\begin{aligned}
& {\left[\begin{array}{l}
i_{\alpha} \\
i_{\beta}
\end{array}\right]=\left[\begin{array}{ccc}
\frac{2}{3} & -\frac{1}{3} & -\frac{1}{3} \\
0 & \frac{1}{\sqrt{3}} & -\frac{1}{\sqrt{3}}
\end{array}\right]\left[\begin{array}{l}
i_{a} \\
i_{b} \\
i_{c}
\end{array}\right],} \\
& {\left[\begin{array}{l}
v_{\alpha} \\
v_{\beta}
\end{array}\right]=\left[\begin{array}{lrr}
\frac{2}{3} & -\frac{1}{3} & -\frac{1}{3} \\
0 & \frac{1}{\sqrt{3}} & -\frac{1}{\sqrt{3}}
\end{array}\right]\left[\begin{array}{l}
v_{a} \\
v_{b} \\
v_{c}
\end{array}\right],}
\end{aligned}
$$

where $i_{a}, i_{b}, i_{c}, v_{a}, v_{b}$, and $v_{c}$ are the phase currents and voltages in the three phase model and $i_{\alpha}, i_{\beta}, v_{\alpha}$ and $v_{\beta}$ are the phase currents and voltages in the $\alpha-\beta$ frame model (AC frame). The $\alpha-\beta$ frame model of the PMSM [1], [2] is

$$
\begin{aligned}
& \dot{\theta}=\omega \\
& \dot{\omega}=-\frac{3}{2} \frac{p \Phi}{J} i_{\alpha} \sin (p \theta)+\frac{3}{2} \frac{p \Phi}{J} i_{\beta} \cos (p \theta)-\frac{B}{J} \omega-\frac{\tau_{l}}{J} \\
& \dot{i}_{\alpha}=-\frac{R}{L} i_{\alpha}+\frac{p \Phi}{L} \omega \sin (p \theta)+\frac{1}{L} v_{\alpha} \\
& \dot{i}_{\beta}=-\frac{R}{L} i_{\beta}-\frac{p \Phi}{L} \omega \cos (p \theta)+\frac{1}{L} v_{\beta}
\end{aligned}
$$

where $R$ is the resistance, $L$ is the inductance, $\Phi$ is the permanent magnet linkage, $B$ is the motor viscous friction constant, $J$ is the rotor inertia, $\theta$ is the rotor position, $\omega$ is the rotor velocity, $p$ is the number of pairs of rotor poles, and $\tau_{l}$ is the load torque. In the $\alpha-\beta$ frame model of PMSM (2), the dynamics of $\theta$ and $\omega$ is mechanical dynamics and the dynamics of $i_{\alpha}$ and $i_{\beta}$ is electrical dynamics. $\theta^{d}$ is the reference position.

\section{B. Review of the Control Method Using the $D Q$ Transformation}

For the simplification of the PMSM model (2), the DQ transformation [15] is

$$
\begin{aligned}
& {\left[\begin{array}{l}
i_{d} \\
i_{q}
\end{array}\right]=\left[\begin{array}{cc}
\cos \left(N_{r} \theta\right) & \sin \left(N_{r} \theta\right) \\
-\sin \left(N_{r} \theta\right) & \cos \left(N_{r} \theta\right)
\end{array}\right]\left[\begin{array}{c}
i_{\alpha} \\
i_{\beta}
\end{array}\right],} \\
& {\left[\begin{array}{l}
v_{d} \\
v_{q}
\end{array}\right]=\left[\begin{array}{cc}
\cos \left(N_{r} \theta\right) & \sin \left(N_{r} \theta\right) \\
-\sin \left(N_{r} \theta\right) & \cos \left(N_{r} \theta\right)
\end{array}\right]\left[\begin{array}{l}
v_{\alpha} \\
v_{\beta}
\end{array}\right]}
\end{aligned}
$$

where $i_{d}$ and $i_{q}$ are the direct and quadrature currents, $v_{d}$ and $v_{q}$ are the direct and quadrature voltages, respectively. This has been widely used for various control methods. Using DQ transformation gives us the DQ frame model of PMSM as

$$
\begin{aligned}
& \dot{\theta}=\omega \\
& \dot{\omega}=\frac{3}{2} \frac{p \Phi}{J} i_{q}-\frac{B}{J} \omega-\frac{\tau_{l}}{J} \\
& \dot{i}_{d}=-\frac{R}{L} i_{d}+\omega i_{q}+\frac{1}{L} v_{d} \\
& \dot{i}_{q}=-\frac{R}{L} i_{q}-\omega i_{d}-\frac{\Phi}{L} \omega+\frac{1}{L} v_{q} .
\end{aligned}
$$

A block diagram of the general control method using the DQ 


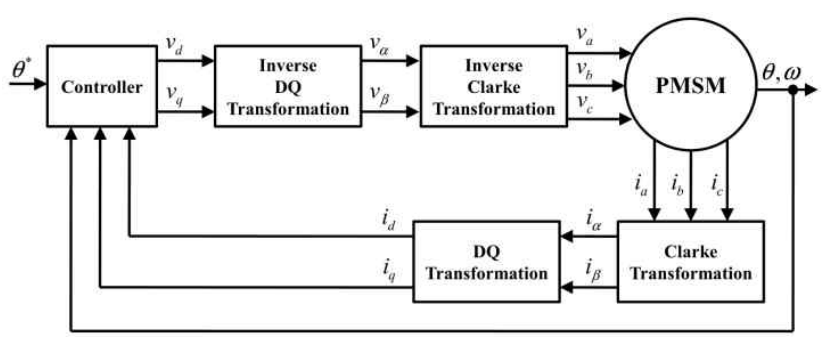

Fig. 1. Block diagram of the general control method using DQ transformation.

transformation is shown in Fig. 1. In Fig. $1, \theta^{*}$ is the reference position. As shown in Fig. 1, using the DQ transformation results in using the inverse DQ transformation. If the controller is designed without the DQ transformation, the structure of the system may be simple.

\section{CONTROLlER DESIGN}

In this Section, we develop the position controller for the PMSM. The proposed controller consists of a mechanical controller, a commutation scheme, an electrical controller, and a load torque observer.

\section{A. Torque Generator}

In this subsection, it is assumed that the load torque $\tau$ is a known constant, and that $i_{\alpha}$ and $i_{\beta}$ are the inputs. In the mechanical dynamics, the main cause of the offset error of the position tracking is the load torque. Thus we design the mechanical controller to compensate for the load torque and eliminate the steady-state error. We define the mechanical tracking error $e_{A}=\left[e_{0} e_{1} e_{2}\right]^{T}$ as

$$
\begin{aligned}
& e_{0}=\int_{0}^{t} e_{1} d t \\
& e_{1}=\theta^{*}-\theta \\
& e_{2}=\omega^{*}-\omega
\end{aligned}
$$

where $\omega^{*}=\dot{\theta}^{*}$ is the reference velocity. The dynamics of the mechanical tracking error is

$$
\begin{aligned}
& \dot{e}_{0}=e_{1} \\
& \dot{e}_{1}=e_{2} \\
& \dot{e}_{2}=\dot{\omega}^{*}+\frac{3}{2} \frac{p \Phi}{J} i_{\alpha} \sin (p \theta)-\frac{3}{2} \frac{p \Phi}{J} i_{\beta} \cos (p \theta)+\frac{B}{J} \omega+\frac{\tau_{l}}{J}
\end{aligned}
$$

In (6), the term $\frac{3}{2} \frac{p \Phi}{J}\left(-i_{\alpha} \sin (p \theta)+i_{\beta} \cos (p \theta)\right)$ is the torque. Thus we can change the term $\frac{3}{2} \frac{p \Phi}{J}\left(-i_{\alpha} \sin (p \theta)+i_{\beta} \cos (p \theta)\right)$ into the PMSM torque, $\tau$. Note that the actual input in the PMSM (2) is not the currents but the voltage. However, we assume that $i_{\alpha}$ and $i_{\beta}$ are the input. Therefore, we define the desired torque as $\tau^{*}$. Then (6) is changed into

$$
\begin{aligned}
& \dot{e}_{0}=e_{1} \\
& \dot{e}_{1}=e_{2} \\
& \dot{e}_{2}=\dot{\omega}^{*}+\frac{\tau^{*}}{J}+\frac{B}{J} \omega+\frac{\tau_{l}}{J} .
\end{aligned}
$$

We design the mechanical controller as

$$
\tau^{*}=J k_{0} e_{0}+J k_{1} e_{1}+J k_{2} e_{2}+J \dot{\omega}^{*}+B \omega+\tau_{l}
$$

where $k_{0}, k_{1}$, and $k_{2}$ are the control gains. Then, the dynamics of the mechanical tracking error (7) becomes

$$
\underbrace{\left[\begin{array}{c}
\dot{e}_{0} \\
\dot{e}_{1} \\
\dot{e}_{2}
\end{array}\right]}_{\dot{e}_{A}}=\underbrace{\left[\begin{array}{ccc}
0 & 1 & 0 \\
0 & 0 & 1 \\
-k_{0} & -k_{1} & -k_{2}
\end{array}\right]}_{A_{1}} \underbrace{\left[\begin{array}{l}
e_{0} \\
e_{1} \\
e_{2}
\end{array}\right]}_{e_{A}} .
$$

If $k_{0}, k_{1}$, and $k_{2}$ are chosen such that $A_{1}$ is Hurwitz, then the origin of the mechanical tracking error dynamics (9) is exponentially stable. The role of the torque generator is the tracking of both position and velocity. In (7) and (8), the position controller (8) makes the position error $e_{1}$ and the speed error $e_{2}$ converge to zero. That is, the torque generator includes both position and speed controllers.

\section{B. Commutation Scheme}

In the electrical dynamics, the currents are the actual inputs. Thus in order to generate the torque, we define the commutation scheme

$$
\begin{aligned}
& i_{\alpha}^{*}=\frac{2 \tau^{*}}{3 p \Phi} \cos \left(p \theta^{d}\right) \\
& i_{\beta}^{*}=\frac{2 \tau^{*}}{3 p \Phi} \sin \left(p \theta^{d}\right)
\end{aligned}
$$

where $\theta^{d}$ is the desired position to generate the desired torque $\tau^{*}$ obtained by (8), and $i_{\alpha}^{*}$ and $i_{\beta}^{*}$ are the desired currents.

Note that the desired position $\theta^{d}$ is different from the position reference $\theta^{*}$. To obtain FOC, we define $\theta^{d}$ as $\theta+\pi / 2$. Then,

$$
\begin{aligned}
& i_{\alpha}^{*}=\frac{2 \tau^{*}}{3 p \Phi} \cos \left(p \theta^{d}\right)=-\frac{2 \tau^{*}}{3 p \Phi} \sin (p \theta) \\
& i_{\beta}^{*}=\frac{2 \tau^{*}}{3 p \Phi} \sin \left(p \theta^{d}\right)=\frac{2 \tau^{*}}{3 p \Phi} \cos (p \theta)
\end{aligned}
$$

The commutation scheme (11) results in field oriented control since the desired direct current $i_{d}^{*}$ and the desired quadrature current $i_{q}^{*}$ become zero and the torque multiplied by the constant as follows

$$
\begin{aligned}
i_{d}^{*} & =\cos (p \theta) i_{\alpha}^{*}+\sin (p \theta) i_{\beta}^{*} \\
& =-\frac{2 \tau^{*}}{3 p \Phi} \sin (p \theta) \cos (p \theta)+\frac{2 \tau^{*}}{3 p \Phi} \cos (p \theta) \sin (p \theta) \\
& =0 \\
i_{q}^{*} & =-\sin (p \theta) i_{\alpha}^{*}+\cos (p \theta) i_{\beta}^{*} \\
& =\frac{2 \tau^{*}}{3 p \Phi}
\end{aligned}
$$




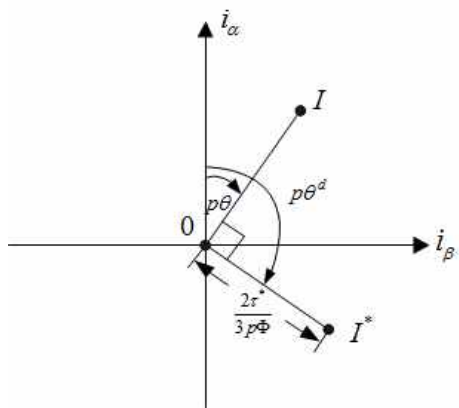

Fig. 2. Illustration of the commutation scheme.

The mean of the commutation scheme (11) is illustrated in Fig. 2. Point $\mathrm{I}\left(i_{\alpha}, i_{\beta}\right)$ is the present current vector of the PMSM while $I^{*}\left(i_{\alpha}^{*}, i_{\beta}^{*}\right)$ is the desired current vectors generated by the commutation scheme (11). $p \theta^{d}$ is always ahead of the present electrical position $p \theta$ by $\pi / 2$. The relationship between $p \theta^{d}$ and $p \theta$ is often referred to as the optimal lead-angle [16]. The magnitude of $I^{*}$ indicates the desired torque for the position tracking.

\section{Electrical Controller}

The actual input of the PMSM (2) is not the currents but the voltage. It is important to guarantee the desired currents for the generating the desired torque. In the current tracking, a DC current offset may appear due to the nonlinearity of the PWM driver. Therefore, we propose the electrical controller to guarantee the desired currents and the compensation of the offset error. The electrical tracking error $e_{B}=\left[e_{3} e_{4} e_{5} e_{6}\right]^{T}$ is defined as

$$
\begin{aligned}
& e_{3}=\int_{0}^{t} e_{4} d t \\
& e_{4}=i_{\alpha}^{*}-i_{\alpha} \\
& e_{5}=\int_{0}^{t} e_{6} d t \\
& e_{6}=i_{\beta}^{*}-i_{\beta}
\end{aligned}
$$

Then we obtain the electrical tracking error dynamics as

$$
\begin{aligned}
& \dot{e}_{3}=e_{4} \\
& \dot{e}_{4}=i_{\alpha}^{*}+\frac{R}{L} i_{\alpha}-\frac{p \Phi}{L} \omega \sin (p \theta)-\frac{1}{L} v_{\alpha} \\
& \dot{e}_{5}=e_{6} \\
& \dot{e}_{6}=i_{\beta}^{*}+\frac{R}{L} i_{\beta}+\frac{p \Phi}{L} \omega \cos (p \theta)-\frac{1}{L} v_{\beta} .
\end{aligned}
$$

For the stability of the electrical tracking error dynamics (14), the electrical dynamics controller is designed by

$$
\begin{aligned}
& v_{\alpha}=L i_{\alpha}^{*}+R i_{\alpha}-p \Phi \omega \sin (p \theta)+L\left(k_{3} e_{3}+k_{4} e_{4}\right) \\
& v_{\beta}=L i_{\beta}^{*}+R i_{\beta}+p \Phi \omega \cos (p \theta)+L\left(k_{3} e_{5}+k_{4} e_{6}\right)
\end{aligned}
$$

where $k_{3}$ and $k_{4}$ are the control gain. With (15), the electrical tracking dynamics becomes

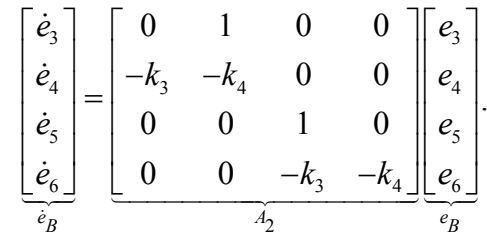

If $k_{3}$ and $k_{4}$ are chosen such that $A_{2}$ is Hurwitz, then the origin of the electrical tracking error dynamics (16) is exponentially stable.

\section{Velocity and Load Torque Observer}

In this subsection, the mechanical controller (8) was designed under the assumption that the speed and the load torque are known. However, it is difficult to know the exact velocity and load torque. Therefore, the speed and load torque observer is designed to estimate the load torque by

$$
\begin{aligned}
\dot{\hat{\theta}}= & \hat{\omega}+l_{1}(\theta-\hat{\theta}) \\
\dot{\hat{\omega}}= & -\frac{3}{2} \frac{p \Phi}{J} i_{\alpha} \sin (p \theta)+\frac{3}{2} \frac{p \Phi}{J} i_{\beta} \cos (p \theta)-\frac{B}{J} \hat{\omega}-\frac{\hat{\tau}_{l}}{J} \\
& +l_{2}(\theta-\hat{\theta}) \\
\dot{\hat{\tau}}_{l}= & l_{3}(\theta-\hat{\theta})
\end{aligned}
$$

where $\hat{\theta}, \hat{\omega}$ and $\hat{\tau}_{l}$ are the estimated position, velocity and load torque, respectively. The estimation errors are defined as

$$
\tilde{x}=\left[\begin{array}{c}
\tilde{\theta} \\
\tilde{\omega} \\
\tilde{\tau}_{l}
\end{array}\right]=\left[\begin{array}{c}
\theta-\hat{\theta} \\
\omega-\hat{\omega} \\
\tau_{l}-\hat{\tau}_{l}
\end{array}\right] .
$$

The estimation error dynamics is

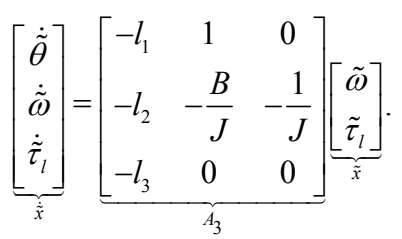

If $l_{1}, l_{2}$ and $l_{3}$ are chosen such that $A_{3}$ is Hurwitz, then the origin of the estimation error dynamics (19) is exponentially stable.

\section{E. Closed-loop System Stability Analysis}

The mechanical controller (8) was designed under the assumptions that the load torque is known and the currents are the input. Therefore, the closed-loop stability that includes the mechanical tracking error dynamics (7), the electrical tracking error dynamics (16), and the estimation error dynamics (19) should be proven. From (7), (16), and (19), the closed-loop system is obtained by 


$$
\begin{aligned}
& \underbrace{\left[\begin{array}{c}
\dot{e}_{0} \\
\dot{e}_{1} \\
\dot{e}_{2}
\end{array}\right]}_{\dot{e}_{A}}=\underbrace{\left[\begin{array}{ccc}
0 & 1 & 0 \\
0 & 0 & 1 \\
-k_{0} & -k_{1} & -k_{2}
\end{array}\right]}_{A_{1}} \underbrace{\left[\begin{array}{l}
e_{0} \\
e_{1} \\
e_{2}
\end{array}\right]}_{e_{A}}
\end{aligned}
$$

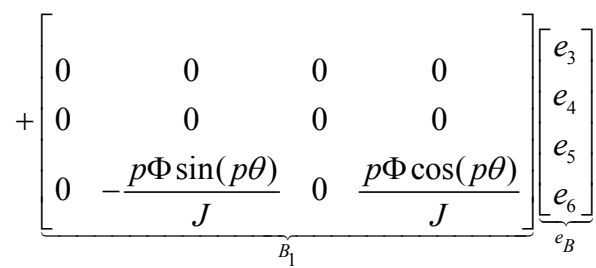

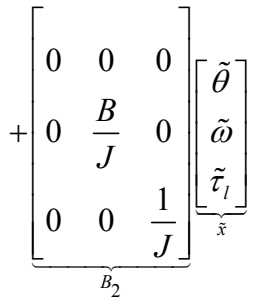

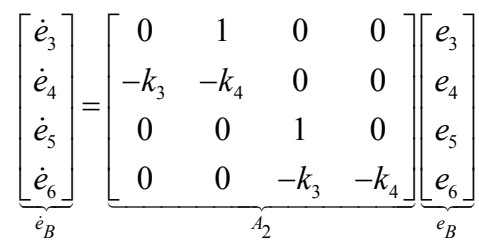

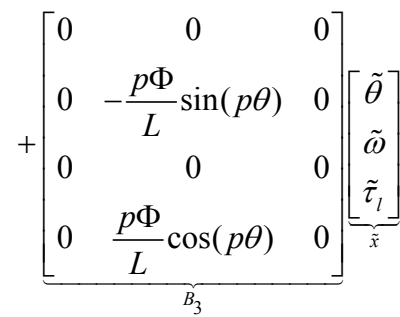

$$
\begin{aligned}
& \underbrace{\left[\begin{array}{c}
\dot{\tilde{\omega}} \\
\dot{\tilde{\tau}}_{l}
\end{array}\right]}_{A_{3}}=\underbrace{\left[\begin{array}{ccc}
-l_{1} & 1 & 0 \\
-l_{2} & -\frac{B}{J} & -\frac{1}{J} \\
-l_{3} & 0 & 0
\end{array}\right]}_{\tilde{\tilde{x}}} \underbrace{\left[\begin{array}{c}
\tilde{\theta} \\
\tilde{\omega} \\
\tilde{\tau}_{l}
\end{array}\right]}_{\tilde{x}}
\end{aligned}
$$

The closed-loop system can be rewritten as

$$
\begin{aligned}
& \dot{e}_{A}=A_{1} e_{A}+B_{1} e_{B}+B_{2} \tilde{x} \\
& \dot{e}_{B}=A_{2} e_{B}+B_{3} \tilde{x} \\
& \dot{\tilde{x}}=A_{3} \tilde{x} .
\end{aligned}
$$

The controller gains are chosen such that $A_{1}, A_{2}$, and $A_{3}$ are Hurwitz. And $B_{1}, B_{2}$ and $B_{3}$ are bounded. Therefore, the dynamics of $e_{A}$ is bounded-input bounded-output (BIBO) stable [16].

\section{F. Optimal Control for Gain Tuning Guide}

In subsection $\mathrm{E}$, it was proven that the control gains of the mechanical controller and the electrical controller can be independently chosen. In order to get the robustness to parameter uncertainties and the gain tuning guide, the LQR method is applied to the proposed method.
First, we apply the LQR method to the mechanical controller. The mechanical error dynamics (9) can be written as

$$
\underbrace{\left[\begin{array}{c}
\dot{e}_{0} \\
\dot{e}_{1} \\
\dot{e}_{2}
\end{array}\right]}_{\dot{e}_{A}}=(\underbrace{\left[\begin{array}{ccc}
0 & 1 & 0 \\
0 & 0 & 1 \\
0 & 0 & 0
\end{array}\right]}_{A_{1_{o p t}}}-\underbrace{\left[\begin{array}{c}
0 \\
0 \\
0
\end{array}\right]}_{B_{1}} \underbrace{\left[\begin{array}{lll}
k_{0} & k_{1} & k_{2}
\end{array}\right]}_{K_{1}}) \underbrace{\left[\begin{array}{c}
e_{0} \\
e_{1} \\
e_{2}
\end{array}\right]}_{e_{A}} .
$$

In (22), the term $-K_{1_{\text {opt }}} e_{A} \quad$ can be regarded as the input as

$$
u_{A}=-K_{1_{\text {opt }}} e_{A}
$$

Thus, (22) becomes

$$
\underbrace{\left[\begin{array}{c}
\dot{e}_{0} \\
\dot{e}_{1} \\
\dot{e}_{2}
\end{array}\right]}_{\dot{e}_{A}}=\underbrace{\left[\begin{array}{ccc}
0 & 1 & 0 \\
0 & 0 & 1 \\
0 & 0 & 0
\end{array}\right]}_{A_{1_{\text {opt }}}} \underbrace{\left[\begin{array}{c}
e_{0} \\
e_{1} \\
e_{2}
\end{array}\right]}_{e_{A}}+\underbrace{\left[\begin{array}{c}
0 \\
0 \\
1
\end{array}\right]}_{B_{1}} u_{A} .
$$

Since the mechanical error dynamics (24) is controllable, the mechanical error dynamics can be applied to the LQR method. The cost function is defined as

$$
J_{A}=\int_{0}^{t}\left(e_{A}^{T} Q_{A} e_{A}+u_{A}^{T} R_{A} u_{A}\right) d t .
$$

To obtain the control gain for optimal control, the Riccati equation is

$$
\begin{aligned}
& A_{1_{\text {opt }}^{T}}^{T} P_{A}+P_{A} A_{1_{\text {opt }}}-P_{A} B_{1_{\text {opt }}} B_{1_{\text {opt }}}^{T} P_{A}+Q_{A}=0 \\
& K_{1_{\text {opt }}}=-R_{A}^{-1} B_{1_{\text {opt }}}^{T} P_{A}
\end{aligned}
$$

Next, we apply the LQR method to the electrical controller. The electrical error dynamics (16) can be written as

$$
\begin{aligned}
& \underbrace{\left[\begin{array}{c}
\dot{e}_{3} \\
\dot{e}_{4}
\end{array}\right]}_{\dot{e}_{B_{1}}}=(\underbrace{\left[\begin{array}{ll}
0 & 1 \\
0 & 0
\end{array}\right]}_{A_{2}}-\underbrace{\left[\begin{array}{c}
0 \\
1
\end{array}\right]}_{B_{2}} \underbrace{\left[\begin{array}{ll}
k_{3} & k_{4}
\end{array}\right]}_{K_{2}}) \underbrace{\left[\begin{array}{c}
e_{3} \\
e_{4}
\end{array}\right]}_{e_{B_{1}}} \\
& \underbrace{\left[\begin{array}{l}
\dot{e}_{5} \\
\dot{e}_{6}
\end{array}\right]}_{\dot{e}_{B_{2}}}=(\underbrace{\left[\begin{array}{ll}
0 & 1 \\
0 & 0
\end{array}\right]}_{A_{2}}-\underbrace{\left[\begin{array}{c}
0 \\
1
\end{array}\right]}_{B_{2}} \underbrace{\left[\begin{array}{ll}
k_{3} & k_{4}
\end{array}\right]}_{K_{2_{o p t}}}) \underbrace{\left[\begin{array}{l}
e_{5} \\
e_{6}
\end{array}\right]}_{e_{B_{2}}} .
\end{aligned}
$$

In (27), the terms $-K_{2_{\text {opt }}} e_{B_{1}}$ and $-K_{2_{\text {opt }}} e_{B_{2}}$ can be regarded as the input as follows

$$
\begin{aligned}
& u_{B_{1}}=-K_{2_{o p t}} e_{B_{1}} \\
& u_{B_{2}}=-K_{2_{o p t}} e_{B_{2}}
\end{aligned}
$$

Thus, (28) becomes 


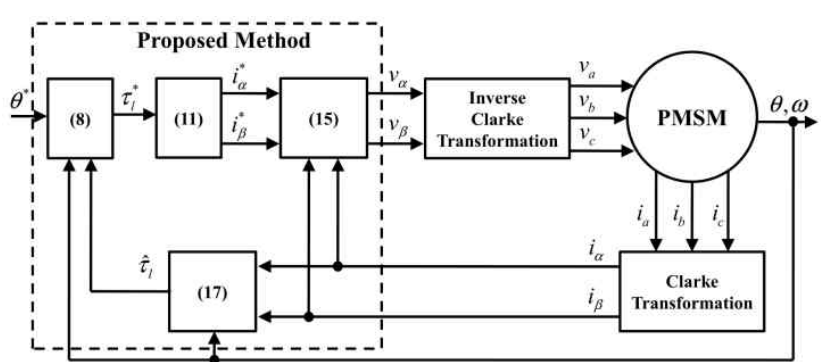

Fig. 3. Block diagram of proposed method

$$
\begin{aligned}
& \underbrace{\left[\begin{array}{c}
\dot{e}_{3} \\
\dot{e}_{4}
\end{array}\right]}_{\dot{e}_{B_{1}}}=\underbrace{\left[\begin{array}{ll}
0 & 1 \\
0 & 0
\end{array}\right]}_{A_{2}} \underbrace{\left[\begin{array}{l}
e_{3} \\
e_{4}
\end{array}\right]}_{e_{B_{1}}}+\underbrace{\left[\begin{array}{l}
0 \\
1
\end{array}\right]}_{B_{2} \text { opt }} u_{B_{1}} \\
& \underbrace{\left[\begin{array}{c}
\dot{e}_{5} \\
\dot{e}_{6}
\end{array}\right]}_{\dot{e}_{B_{2}}}=\underbrace{\left[\begin{array}{ll}
0 & 1 \\
0 & 0 p t
\end{array}\right]}_{A_{2}} \underbrace{\left[\begin{array}{l}
e_{5} \\
e_{6}
\end{array}\right]}_{e_{B_{2}}}+\underbrace{\left[\begin{array}{c}
0 \\
1
\end{array}\right]}_{B_{2_{o p t}}} u_{B_{2}} .
\end{aligned}
$$

Since the electrical error dynamics (29) is controllable, the electrical error dynamics can be applied to the LQR method. The cost function is defined as

$$
\begin{aligned}
& J_{B_{1}}=\int_{0}^{t}\left(e_{B_{1}}^{T} Q_{B} e_{B_{1}}+u_{B_{1}}^{T} R_{B} u_{B_{1}}\right) d t \\
& J_{B_{2}}=\int_{0}^{t}\left(e_{B_{2}}^{T} Q_{B} e_{B_{2}}+u_{B_{2}}^{T} R_{B} u_{B_{2}}\right) d t .
\end{aligned}
$$

To obtain the control gain for optimal control, the Riccati equation is

$$
\begin{aligned}
& A_{2_{\text {opt }}^{T}}^{T} P_{B}+P_{B} A_{2_{\text {opt }}}-P_{B} B_{2_{\text {opt }}} B_{2_{\text {opt }}^{T}}^{T} P_{B}+Q_{B}=0 \\
& K_{2_{\text {opt }}}=-R_{B}^{-1} B_{2_{\text {opt }}^{T}}^{T} P_{B}
\end{aligned}
$$

Fig. 3 shows the block diagrams of the proposed method. The load torque observer (17) estimates the load torque. Then the mechanical controller (8) generates the desired torque $\tau^{*}$ using the position $\theta$, the velocity $\omega$, and the estimated load torque $\tau_{l}$. The desired currents $i_{\alpha}^{*}$ and $i_{\beta}^{*}$ are made by the commutation scheme (10). Then, the input voltages are made by the electrical controller (15).

\section{SIMULATION AND EXPERIMENTAL RESULTS}

Simulations and experiments were performed to evaluate the performance of the proposed controller. The PMSM in the active protective launcher system shown in Fig. 4 was used. PMSM is used to rotate the gun barrel in an active protective launcher system. The requirement of the position control is that the absolute final position error should be less than $0.0049 \mathrm{rad}$. The required maximum velocity is $2 \pi \mathrm{rad} / \mathrm{s}$. The motor drive used IGBT and its switching frequency was 8 $\mathrm{MHz}$. When the gun barrel is rotated, the load torque appeared. The PMSM parameters are listed in Table 1.

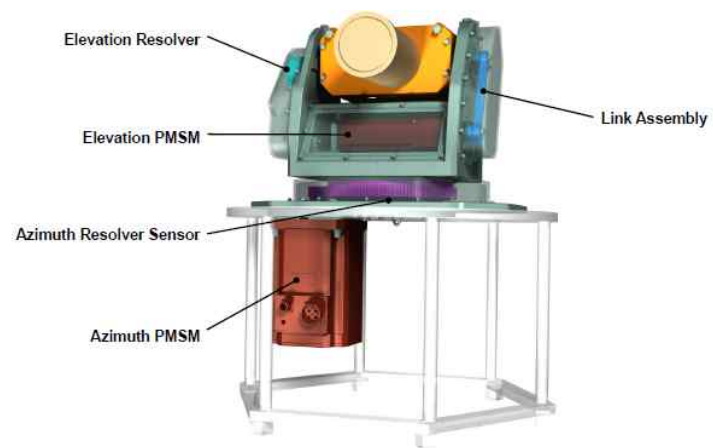

Fig. 4. Active protective launcher system.

TABLE I

PMSM PARAMETERS

\begin{tabular}{|c|c|c|c|}
\hline Para. & Value & Para. & Value \\
\hline$L$ & $11 \mathrm{mH}$ & $R$ & $0.12 \Omega$ \\
\hline$\Phi$ & $0.18 \mathrm{Vsec} / \mathrm{rad}$ & $J$ & $0.006 \mathrm{kgm}^{2}$ \\
\hline$p$ & 3 & $B$ & $0.001 \mathrm{Nms} / \mathrm{rad}$ \\
\hline
\end{tabular}

TABLE II

CONTROL PARAMETERS

\begin{tabular}{|c|c|c|c|}
\hline Para. & Value & Para. & Value \\
\hline$k_{0}$ & 0.7071 & $k_{1}$ & 707.1869 \\
\hline$k_{2}$ & 80.0898 & $k_{3}$ & 1 \\
\hline$k_{4}$ & 316.2306 & $l_{1}$ & 239.8 \\
\hline$l_{2}$ & 1273 & $l_{3}$ & -1000.5 \\
\hline
\end{tabular}

The control gains were obtained using $Q_{A}=\operatorname{diag}(0.5,500000$, 5000), $R_{A}=1, Q_{B}=\operatorname{diag}(1,100000)$ and $R_{B}=1$ as shown in Table 2. The PMSM was designed to move the launcher in the active protective launcher system shown in Fig. 4. In this system, high velocity is not required since the launcher is not moved quickly. Thus, the position reference with slow velocity as shown in Fig 5 was used in these simulations and experiments.

\section{A. Simulation Results}

Simulations were performed to evaluate the estimation performance of the proposed velocity and load torque observer. Figs. 6 and 7 show the estimation performances of the velocity and the load torque. The blue solid lines are the actual velocity and load torque, and the red dashed-lines are estimated velocity and load torque. We see that the estimations of the velocity and the load torque tracked the actual velocity and load torque well.

\section{B. Experimental Results}

Experiments were performed to evaluate the performance of the proposed method. In a comparative study, the conventional PI control as shown in Fig. 8 was executed. For the experiments, the proposed method and the conventional PI control method were coded in C Language using a TMS320F2808. In the position PI controller (PI1), the 


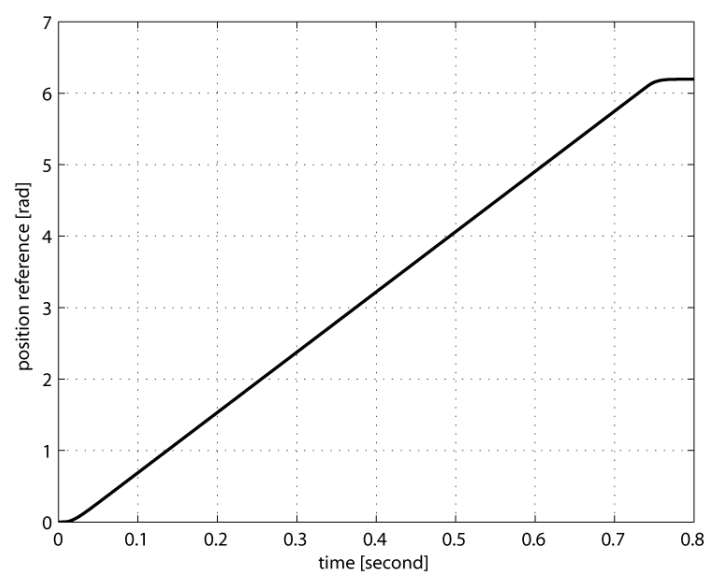

Fig. 5. Position reference.

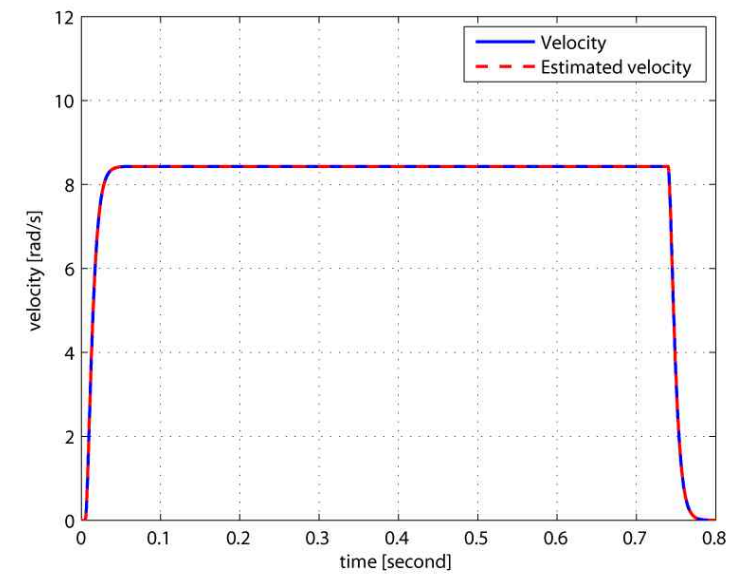

Fig. 6. Estimation performance of the velocity

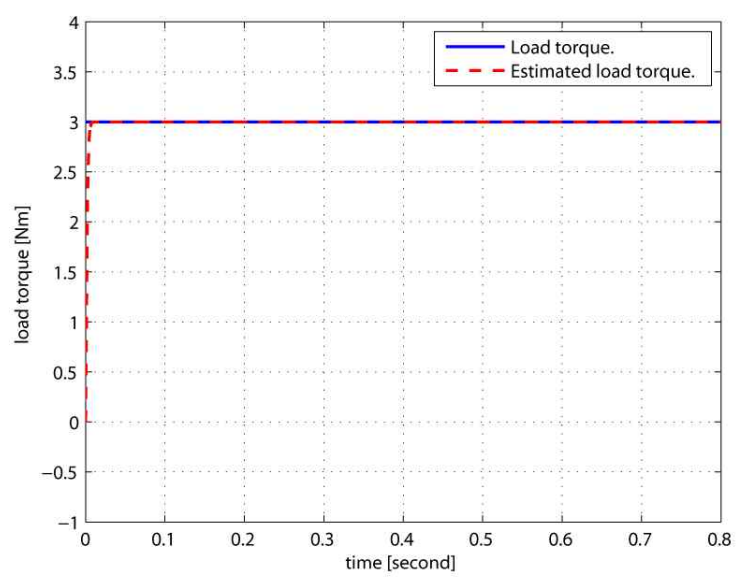

Fig. 7. Estimation performance of the load torque

proportional and integral gains were 1200 and 2, respectively. In the velocity PI controller (PI2), the proportional and integral gains were 100 and 0.7 , respectively. And 400 and 2 were used as the proportional and integral gains in the electrical PI controller (PI3). Since the electrical PI controller controls $i_{q}$, the PI controller for $i_{q}$ can be regarded as the torque controller.

A resolver was used to obtain the position. The currents

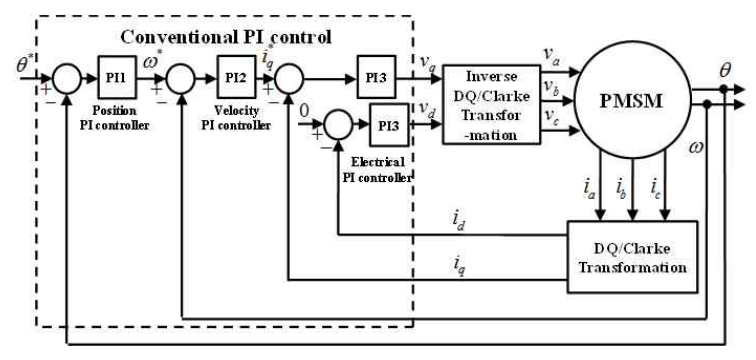

Fig. 8. Block diagram of conventional PI control method.

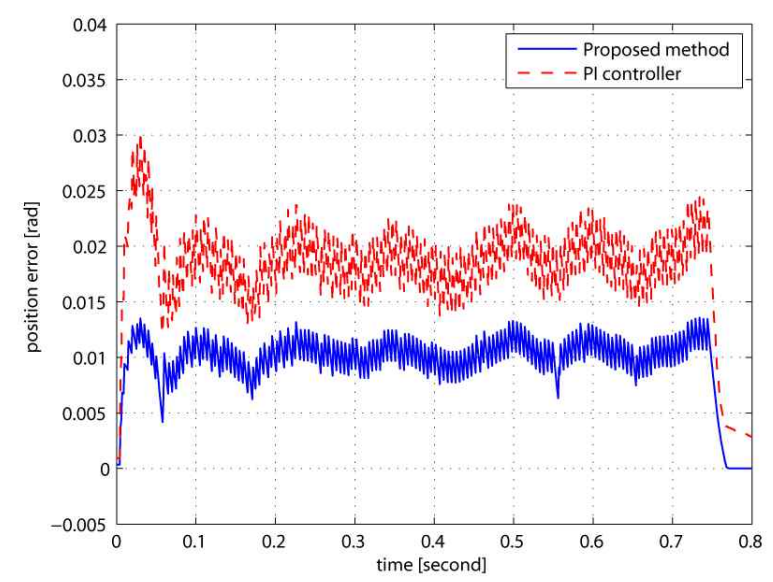

Fig. 9. Position tracking errors of the proposed method and the PI controller.

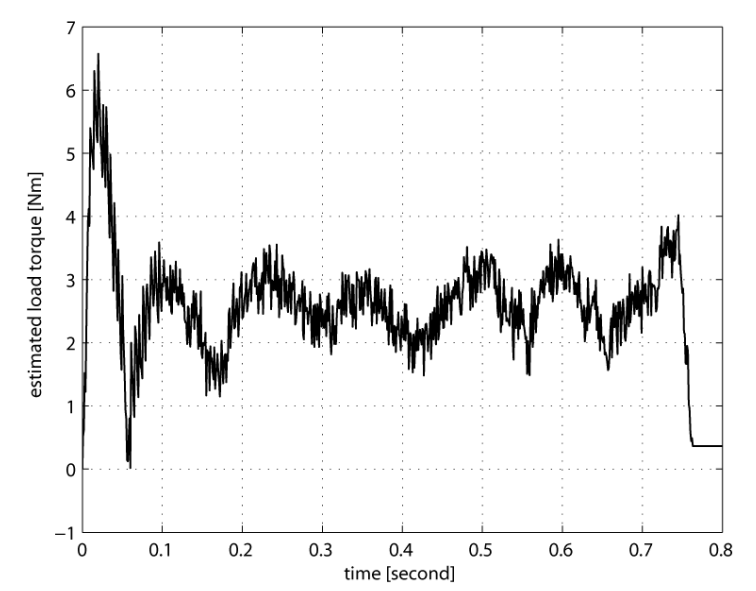

Fig. 10. Estimated load torque.

were measured by the current sensors embedded in the IGBT motor drive. The sampling rate was $5 \mathrm{kHz}$, and 12-bit analog to digital (A/D) converters and 12-bit digital to analog (D/A) converters were used. To validate the robustness of the proposed method against parameter uncertainties, we used the parameters which differed from the actual parameters within $10 \%$ uncertainties.

The position tracking errors of both methods are shown in Fig. 9. The position tracking performances of both methods 


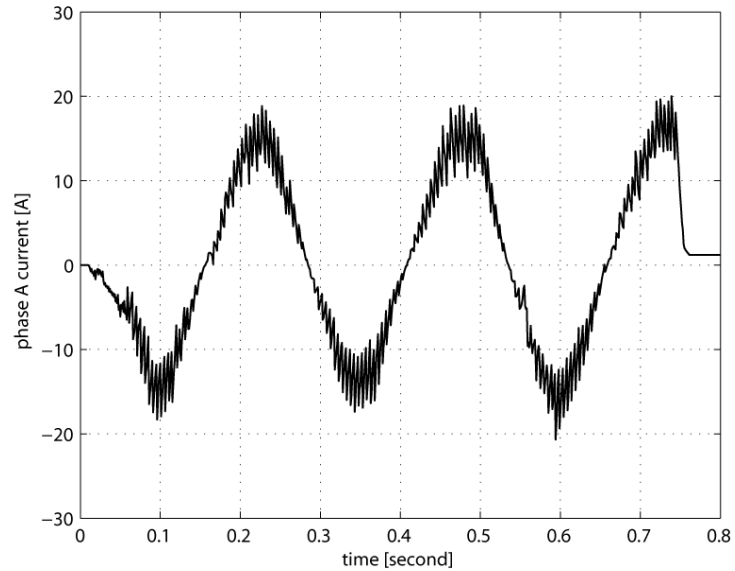

(a) $i_{\alpha}$

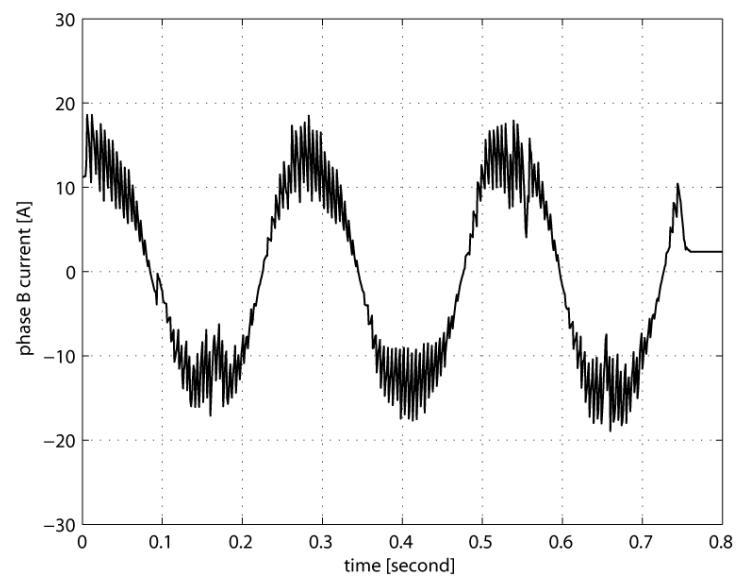

(b) $i_{\beta}$

Fig. 11. Currents in Phase A and B.

satisfied the requirement. Although there were parameter uncertainties and a disturbance, the performance of the proposed method was better than that of the PI controller. Fig. 10 shows the estimated load torque. In the start position, the load torque was increased since the load torque including friction was estimated by the proposed observer. Thus, the position tracking errors of both methods were increased. In the proposed method, the overshoot of the position tracking error was suppressed due to the compensation of the load torque compared to that of the PI controller. The position tracking error in the final position may go to zero by the integral action of PI controller as long as time goes to infinity. However the convergence rate was slow. If the integral gain gets bigger for a fast convergence, the amplified measurement noise may result in a degradation of the performance. In contrast, since the proposed method estimated the load torque as shown in Fig. 10 and compensated for the load torque, the position tracking error of the proposed method in the final position was close to zero. Furthermore, the position ripple was reduced by the proposed method. Since there were ripples due to switching, modeling uncertainty, nonideal sinusoidal flux distribution,

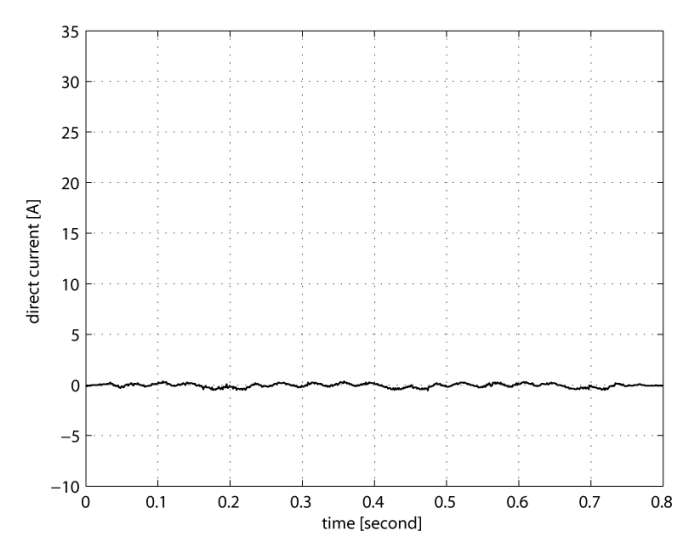

(a) $i_{d}$

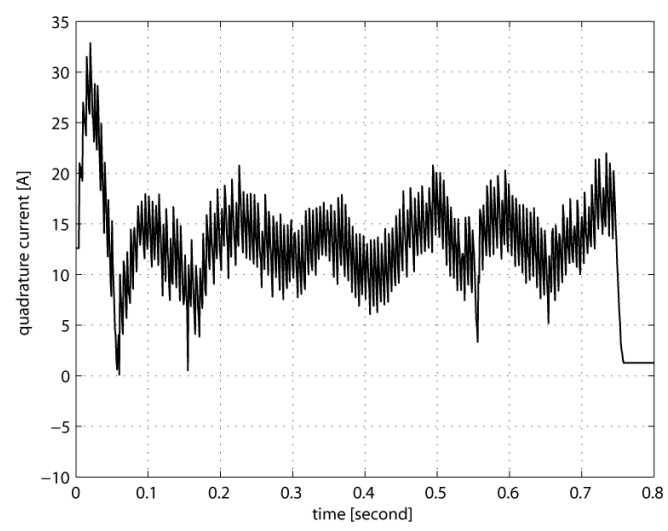

(b) $i_{q}$.

Fig. 12. Direct and quadrature currents.

cogging torque and so on, ripples appeared in the position. The phase A and B currents are shown in Fig. 11. In the initial position, the currents had jerks due to the load torque. Since the final position of the position reference was 1 rotation, i.e., $6.28 \mathrm{rad}$, the currents had 3 cycles. In the currents, unavoidable ripples also appeared due to the same causes in the position case. The direct and quadrature currents are depicted in Fig. 12. As shown in Fig. 12, it was observed that FOC was achieved by the proposed method. The zero direct current was obtained by the proposed method. In the start position, the quadrature current was increased to compensate for load torque that included friction. The quadrature current in the final position was not zero to compensate for the load torque.

\section{CONCLUSIONS}

This paper proposed the robust optimal nonlinear control to compensate for constant disturbances and load torque and to obtain FOC based on the AC frame for the position tracking of PMSMs. The proposed method consists of the torque generator, the commutation scheme, the electrical controller, 
and the load torque observer. The mechanical controller was designed to compensate for the load torque and the offset error and generate the desired torque. And the commutation scheme was proposed to make the desired currents for the desired torque. The electrical controller was developed to guarantee the desired currents. The load torque observer was also designed. In order to get the robustness to parameter uncertainties and the gain tuning guide, the LQR method was applied to the proposed method. We validated that the position tracking performance of the proposed method is superior to that of the conventional method and the FOC was obtained based on AC frame via experiments. It was observed that the proposed method estimated the load torque and compensated for the offset error. The drawback of the proposed method is that full state information is required. This limitation can be overcome by using sensorless control methods [11], [18], [19]

\section{REFERENCES}

[1] B. K. Bose, Power Electronics and AC Drives, Englewood Cliffs, NJ:Prentice-Hall, 1986.

[2] H. A. Toliyat and G. B. Kliman, Handbook of Electric Motors, New York: Marcel Dekker, 2004.

[3] J. Hur, "Characteristic analysis of interior permanentmagnet synchronous motor in electrohydraulic power steering systems," IEEE Trans. Ind. Electron., Vol. 55, No. 6, pp. 2316-2323, Jun. 2008.

[4] B. GrCar, P. Cafuta, M. Znidaric, and F. Gausch, "Nonlinear control of synchronous servo drive," IEEE Trans. Contr. Syst. Technol., Vol. 4, No. 2, pp. 177-184, Mar. 1996.

[5] I.-C. Baik, K.-H. Kim, and M.-J. Youn, "Robust nonlinear speed control of PM synchronous motor using boundary layer integral sliding mode control technique," IEEE Trans. Contr. Syst. Technol., Vol. 8, No. 1, pp.47-54, Jan. 2000.

[6] M.-J. Chung and D.-G. Gweon, "Design optimization and development of linear brushless permanent magnet motor," Int. J. Contr., Automat., and Syst., Vol. 1, No. 3, pp.351-357, 2003.

[7] V. M. Hernandez-Guzman and R. Silva-Ortigoza, "PI control plus electric current loops for PM synchronous motors," IEEE Trans. Contr. Syst. Technol., Vol. 19, No. 4, pp. 868-873, Jul. 2011

[8] F.-J. Lin and C.-H. Lin, "A permanent-magnet synchronous motor servo drive using self-constructing fuzzy neural network controller," IEEE Trans. Energy Conv., Vol. 19, No. 1, pp. 66-72, Mar. 2004.

[9] W. Kim, D. Shin, and C. C. Chung, "The Lyapunov-based controller with a passive nonlinear observer to Improve position tracking performance of microstepping in permanent magnet stepper motors," Automatica, Vol. 48, No. 12, pp. 3064-3074, Dec. 2012.

[10] W. Kim, D. Shin, and C. C. Chung, "Microstepping with nonlinear torque modulation for permanent magnet stepper motors," IEEE Trans. Control Syst. Technol., Vol. 21, No. 5, pp. 1971-1979, Sep. 2013.

[11] J. Solsona, M. I. Valla, and C. Muravchik, "Nonlinear control of a permanent magnet synchronous motor with disturbance torque estimation," IEEE Trans. Energy Conv., Vol. 15, No. 2, pp. 163-168, Jun. 2000.

[12] G. Zhu, A. Kaddouri, and L.-A. Dessaint, and O. Akhrif, "Nonlinear state observer for the sensorless control of a permanent-magnet AC machine," IEEE Trans. Ind. Electron., Vol. 48, No. 6, pp. 1098-1108, Dec. 2001.

[13] M. Ruderman, J. Krettek, F. Hoffmann, and T. Bertram, "Optimal state space control of dc motor," in Proc. IFAC World Congress, pp. 5796-5801, 2008.

[14] C. Olalla, R. Leyva, A. E. Aroudi, and I. Queinnec, "Robust LQR control for PWM converters: an LMI Approach," IEEE Trans. Ind. Electron., Vol. 56, No. 7, pp. 2548-2558, Jul. 2009.

[15] R. H. Park, "Two-reaction theory of synchronous machines generalized method of analysis," AIEE Trans., Vol. 48, pp. 716-727, 1929.

[16] A. Verl and M. Bodson, "Torque maximization for permanent magnet synchronous motors," IEEE Trans. Contr. Syst. Technol., Vol. 6, No. 6, pp. 740-744, Nov. 1998.

[17] H. Khalil, Nonlinear Systems, 3rd ed., Upper Saddle River, NJ:Prentice-Hall, 2002.

[18] M. L. Corradini, G. Ippoliti, S. Longhi, and G. Orlando, “A quasi-sliding mode approach for robust control and speed estimation of PM synchronous motors," IEEE Trans. Ind. Electron., Vol. 59, No. 2, pp. 1096-1104, Feb. 2012.

[19] Z. Qiao, T. Shi, Y. Wang, Y. Yan, C. Xia, and X. He, "New sliding-mode observer for position sensorless control of permanent-magnet synchronous motor," IEEE Trans. Ind. Electron., Vol. 60, No. 2, pp. 710-719, Feb. 2013.

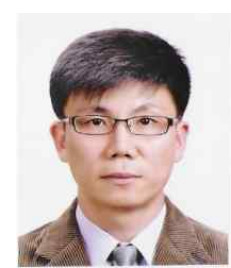

Dong-Hyun Ha received his M.S. in electronic engineering from Soongsil University, Seoul, Korea, in 1995. He is now his Ph.D. candidate in Electrical Engineering from Hanyang University, Seoul, Korea, from 2005. He was a principal research engineer at the Hyundai Mobis R\&D Center from 1995 to 2003. Since 2004, He has work at the Hyundai Rotem Company R\&D Center as a chief research engineer. His current research interests include Vehicle Control System, power converter and power management systems, driving control systems, soft switching techniques for renewable energies and battery chargers.

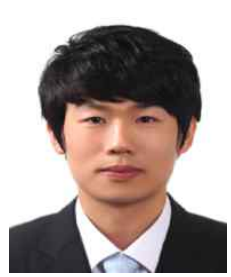

Chang-Soon Lim received his B.S. in electronic engineering from Soongsil University, Seoul, Korea, in 2009, and his M.S. degree in Electrical Engineering from Hanyang University, Seoul, Korea, in 2011, where he is currently working toward his $\mathrm{Ph} . \mathrm{D}$. degree. His current research interests include the modeling and control of power converter systems, soft switching techniques for renewable energies and battery chargers.

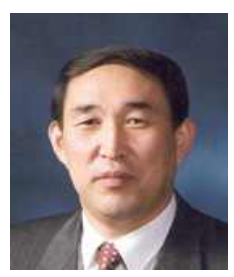

Dong-Seok Hyun received the B.S. and M.S. from Hanyang University, Seoul, Korea, in 1973 and 1978, respectively, and the Ph.D. degree from Seoul National University, Seoul, Korea, in 1986, all in electrical engineering. From 1976 to 1979, he was a Researcher with the Agency of Defense Development, Korea. From 1984 to 1985, he was a Research Associate in the Department of 
Electrical Engineering, University of Toledo, Toledo, OH, USA and from 1988 to 1989, he was a Visiting Professor in the Department of Electrical Engineering, Technical University of Munich, Munich, Germany. Since 1979, he has been with Hanyang University, where he is currently a Professor in the Department of Electrical Engineering. He is the author of more than 650 publications concerning electric machine design, highpower engineering, power electronics, and motor drives. His research interests include power electronics, motor drives, traction, and their control systems. Dr. Hyun is a member of the IEEE Power Electronics, Industrial Electronics, Industry Applications, and Electron Devices Societies. He is also a member of the Institution of Engineering and Technology, the Korean Institute of Power Electronics, and the Korean Institute of Electrical Engineers. 